\title{
FORMATION OF DOUBLE GALAXIES BY TIDAL CAPTURE
}

\author{
S. M. AllADIN, A. POTDAR, and K. S. SASTRY \\ Centre of Advanced Study in Astronomy, Osmania University, Hyderabad, India
}

\begin{abstract}
The conditions under which double galaxies may be formed by tidal capture are considered. Estimates for the increase in the internal energy of colliding galaxies due to tidal effects are used to determine the magnitudes $V_{\text {cap }}$ and $V_{\text {dis }}$ of the maximum relative velocities at infinite separation required for tidal capture and tidal disruption respectively. A double galaxy will be formed by tidal capture without tidal disruption of a component if $V_{\text {cap }}>V_{i}$ and $V_{\text {cap }}>V_{\text {dis }}$ where $V_{i}$ is the initial relative speed of the two galaxies at infinite separation. If the two galaxies are of the same dimension, formation of double galaxies by tidal capture is possible in a close collision either if the two galaxies do not differ much in mass and density distribution or if the more massive galaxy is less centrally concentrated than the other. If we assume, as statistics suggest, that the mass of a galaxy is proportional to the square of its radius, it follows that the probability of the formation of double galaxies by tidal capture increases with the increase in mass of the galaxies and tidal disruption does not occur in a single collision for any distance of closest approach of the two galaxies.
\end{abstract}

We know from classical dynamics that two stars approaching each other from a distance cannot form a binary system without the aid of a third perturbing body. An encounter between two galaxies is, however, an inelastic collision and may lead to the formation of a double galaxy under certain circumstances.

Consider a galaxy of mass $M_{1}$ approaching a galaxy of mass $M_{2}$ from a great distance. Let $U$ be the total internal energy of a galaxy (i.e. the sum of the potential energy of the galaxy and the kinetic energy of the stars constituting the galaxy) and let $E$ be the total external energy of the galaxies (i.e. the sum of the potential energy due to the mutual gravitational attraction of the two galaxies and the kinetic energy due to the orbital motion of the two galaxies). The total energy,

$$
\mathscr{E}=E+U_{1}+U_{2}
$$

is conserved.

The tidal effects of the encounter result in increase in the velocities of the stars in the two galaxies, so that the total internal energy, $U_{1}+U_{2}$ of the galaxies increases at the expense of the external energy $E$. Let $\Delta U_{1}$ and $\Delta U_{2}$ be the increments in the internal energies of the two galaxies due to the encounter, then,

$$
E_{i}=E_{f}+\Delta U_{1}+\Delta U_{2},
$$

where $E_{i}$ and $E_{f}$ are the initial and final values of the external energy.

Following Rood (1965) we define the capture velocity, $V_{\text {cap }}$, as the initial relative velocity at infinite separation for which $E_{f}=0$, so that,

$$
\frac{1}{2} \frac{M_{1} M_{2}}{M_{1}+M_{2}} V_{\text {cap }}^{2}=\Delta U_{1}+\Delta U_{2} .
$$

We have obtained $\Delta U_{1}$ and $\Delta U_{2}$ at various distances of closest approach of the two 
galaxies by selecting 48 stars as representative of the test galaxy and calculating at each instant the tidal force exerted on them by the field galaxy. The tidal force, integrated over all time, gives the change in velocity of the representative stars during the collision, from which the change in the internal energy of the entire galaxy during the collision is inferred.

The following simplifying assumptions have been made in the determination of $\Delta U$ :

(i) The galaxies are spherically symmetric configurations whose density distribution does not change during the encounter. With this assumption it is convenient to represent the density distribution in galaxies by that of a polytrope and to derive the relevant forces from the theory of polytropes as explained in Alladin (1965), Sastry and Alladin (1970), and Potdar and Ballabh (1974). Rood (1965) indicated that the polytrope of index $n=4$ closely represents the density distribution of a typical globular galaxy. Unless otherwise stated this model of density distribution is adopted for the galaxies.

(ii) The relative motion of the two galaxies is considered as uniform rectilinear motion with the distance, $p$, and velocity, $\mathbf{V}_{p}$, at closest approach, same as those for the actual relative orbit of the galaxies. Since the tidal forces due to the collisions are of impulsive nature and much of the gain in kinetic energy of the galaxies occurs when the galaxies are close to each other, this assumption does not lead to much loss of accuracy.

(iii) The motion of the stars in the galaxies is neglected in comparison with the orbital motion of the galaxies during the encounter. The adequacy of this impulsive approximation is discussed in Sastry and Alladin (1970).

With these assumptions and the use of the theory of polytropes, we get:

$$
\Delta U_{2}=\frac{1}{2} \frac{G^{2} M_{1}^{2} M_{2}}{V_{\mathrm{p}}^{2} R_{2}^{2}} J\left(R_{1} / R_{2}, p / R_{2}, n_{1}, n_{2}\right),
$$

where $G$ is the gravitational constant, $R_{1}$ and $R_{2}$ are the radii of the two galaxies represented as polytropes of indices $n_{1}$ and $n_{2}$. $J$ has been obtained numerically as a function of the distance of closest approach, $p$, for various values of $n_{1}$ and $n_{2}$ for $R_{1}=R_{2}$ by Sastry (1973) and for various values of $R_{1} / R_{2}$ for $n_{1}=n_{2}=4$ by Potdar (1974).

If the two galaxies do not interpenetrate each other during the encounter $J$ varies as $\left(R_{2} / p\right)^{4}$ and Equation (4) reduces to the same form as that obtained by Spitzer (1958) for the change in the internal energy of a galactic cluster due to the tidal effects of a passing interstellar cloud.

Under certain circumstances the tidal forces may be so strong that one of the two galaxies may be disrupted during the collision. The condition $\Delta U /|U|=1$ is a convenient order of magnitude discriminant of the disruptive effects of the tidal forces. We shall regard a galaxy to be disrupted if $\Delta U /|U| \geqslant 1$. It should be noted, however, that $\Delta U /|U|=1$ does not necessarily imply total disruption of the galaxy; a significant fraction of its mass may still remain bound in this case. We define the disruption 
velocity, $V_{\text {dis }}$, as that relative velocity at infinite separation before the collision for which $\Delta U$ for one of the galaxies is of the order of its initial internal energy $|U| .|U|$ may be conveniently obtained by Virial theorem from the potential energy of a polytrope.

In order that a double galaxy may be formed by tidal capture without one of the galaxies getting disrupted we require that,

$$
V_{\text {cap }}>V_{i}, \quad V_{\text {cap }}>V_{\text {dis }},
$$

where $\mathbf{V}_{i}$ is the initial relative velocity of the galaxies at infinite separation.

To obtain velocities at infinite separation from the corresponding velocities at closest approach, we make use of the following relation which expresses the conservation of energy:

$$
E_{i}=E_{p}+\left(\Delta U_{1}\right)_{p}+\left(\Delta U_{2}\right)_{p}
$$

The subscript $p$ denotes the value of the quantity at the distance of closest approach. In writing (6) we have assumed that the tidal effects of the collision are symmetrical about the distance of closest approach so that $\left(\Delta U_{1}\right)_{p}+\left(\Delta U_{2}\right)_{p}=\frac{1}{2}\left(\Delta U_{1}+\Delta U_{2}\right)$. Strictly speaking $\left(\Delta U_{1}\right)_{p}+\left(\Delta U_{2}\right)_{p}<\frac{1}{2}\left(\Delta U_{1}+\Delta U_{2}\right)$ since the relative velocity of the galaxies is smaller in the second half of the encounter.

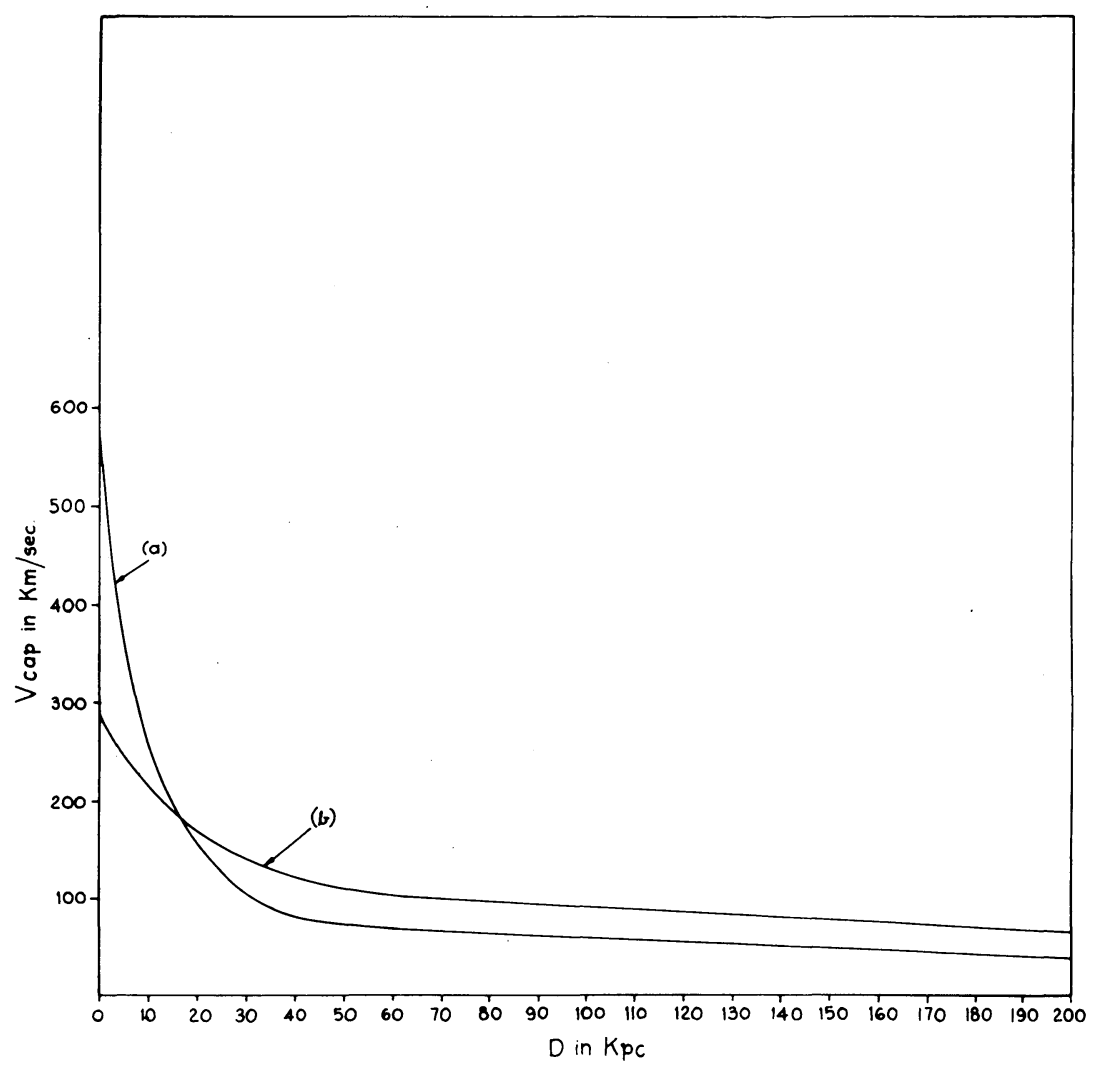

Fig. 1. The run of $V_{\text {cap }}$ with $D$ for cases (a) and (b). 


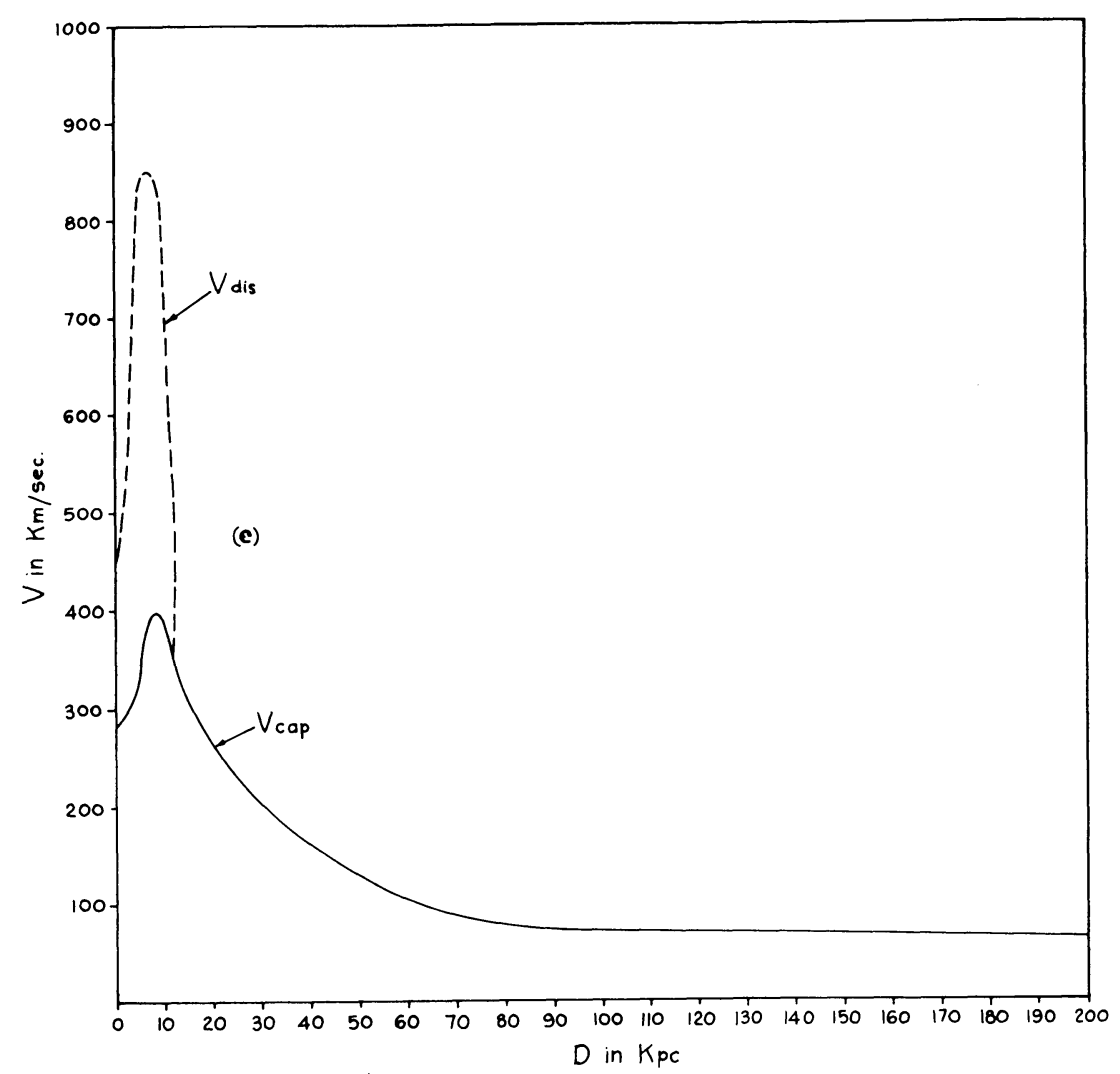

Fig. 2. The run of $V_{\text {cap }}$ and $V_{\text {dis }}$ with $D$ for case (c).

\section{TABLE I}

Impact parameters $D_{c}$ and $D_{d}$ in kpc corresponding to $V_{\text {cap }}$ and $V_{\text {dis }}$ as a function of $p(\mathrm{kpc})$

\begin{tabular}{llllccc}
\hline Case & $p$ & 0 & 2 & 6 & 10 & 20 \\
\hline (a) & $D_{c}$ & 0 & 4.5 & 36 & 60 & 250 \\
(b) & $D_{c}$ & 0 & 3.9 & 13 & 27 & 60 \\
(c) & $D_{c}$ & 0 & 3.6 & 8.3 & 15 & 80 \\
& $D_{d}$ & 0 & 2.7 & 6.8 & 12 & - \\
(d) & $D_{c}$ & 0 & 4.4 & 21 & 57 & 250 \\
& $D_{d}$ & 0 & 2.2 & - & - & - \\
(e) & $D_{c}$ & 0 & 3.7 & 12 & 26 & 60 \\
& $D_{d}$ & 0 & 2.1 & 6.4 & 11 & 30 \\
(f) & $D_{c}$ & 0 & 4.9 & 11 & 20 & 110 \\
(g) & $D_{c}$ & 0 & 19 & 130 & $H^{\text {a }}$ & $H$ \\
(h) & $D_{c}$ & 0 & 26 & $H$ & $H$ & $H$
\end{tabular}

a $H$ denotes values higher than 300 . 
The impact parameter, $D$, has been obtained by assuming that the stars in the galaxies have spherically symmetric distribution of velocities so that from the law of conservation of angular momentum we have:

$$
D V_{i}=p V_{p} \text {. }
$$

The capture velocities are given as a function of the impact parameter for encounters between galaxies of same dimension in Figures 1 to 5 . The disruption velocities are also indicated whenever $V_{\text {dis }}>V_{\text {cap }}$. The values of the impact parameter corresponding to various values of $p$ for capture and disruption cases are given in Table I. $D_{c}$ and $D_{d}$ denote the values of $D$ for collisions with velocities $V_{\text {cap }}$ and $V_{\text {dis }}$ respectively.

The following cases are considered:
(a) $\quad R_{1}=R_{2}=10 \mathrm{kpc}$,
$M_{1}=M_{2}=10^{11} M_{\odot}$,
$n_{1}=n_{2}=4$
(b) $\quad R_{1}=R_{2}=10 \mathrm{kpc}$,
$M_{1}=M_{2}=10^{11} M_{\odot}$,
$n_{1}=n_{2}=0$
(c) $R_{1}=R_{2}=10 \mathrm{kpc}$,
$M_{1}=M_{2}=10^{11} M_{\odot}$,
$n_{1}=4, \quad n_{2}=0$
(d) $R_{1}=R_{2}=10 \mathrm{kpc}$,
$M_{1}=10^{12} M_{\odot}$
$M_{2}=10^{11} M_{\odot}$,
$n_{1}=n_{2}=4$
(e) $\quad R_{1}=R_{2}=10 \mathrm{kpc}$,
$M_{1}=10^{12} M_{\odot}$
$M_{2}=10^{11} M_{\odot}$,
$n_{1}=n_{2}=0$
(f)
$R_{1}=R_{2}=10 \mathrm{kpc}$
$M_{1}=10^{12} M_{\odot}$,
$M_{2}=10^{11} M_{\odot}, n_{1}=0, n_{2}=4$.

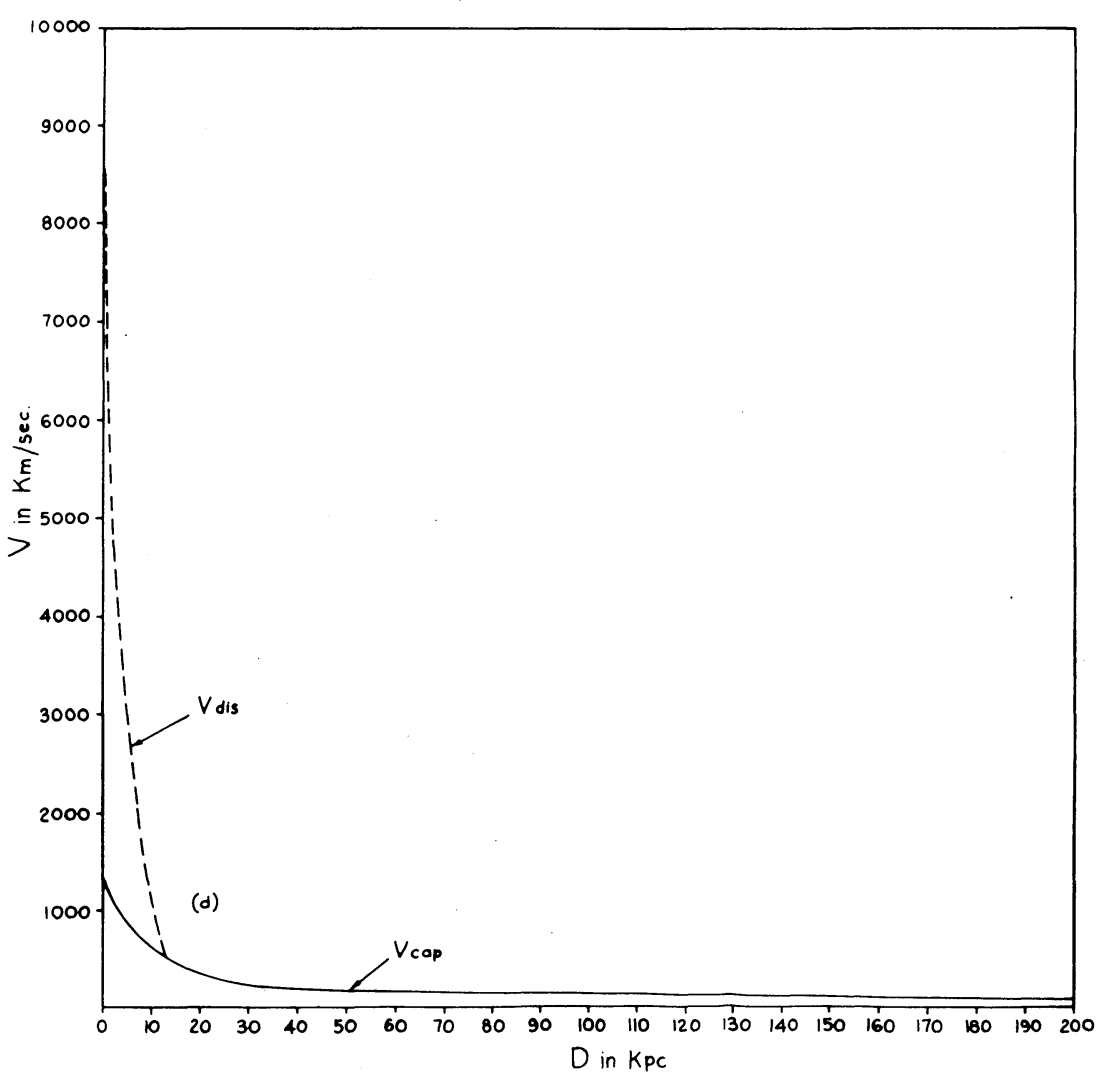

Fig. 3. The run of $V_{\text {cap }}$ and $V_{\text {dis }}$ with $D$ for case (d). 


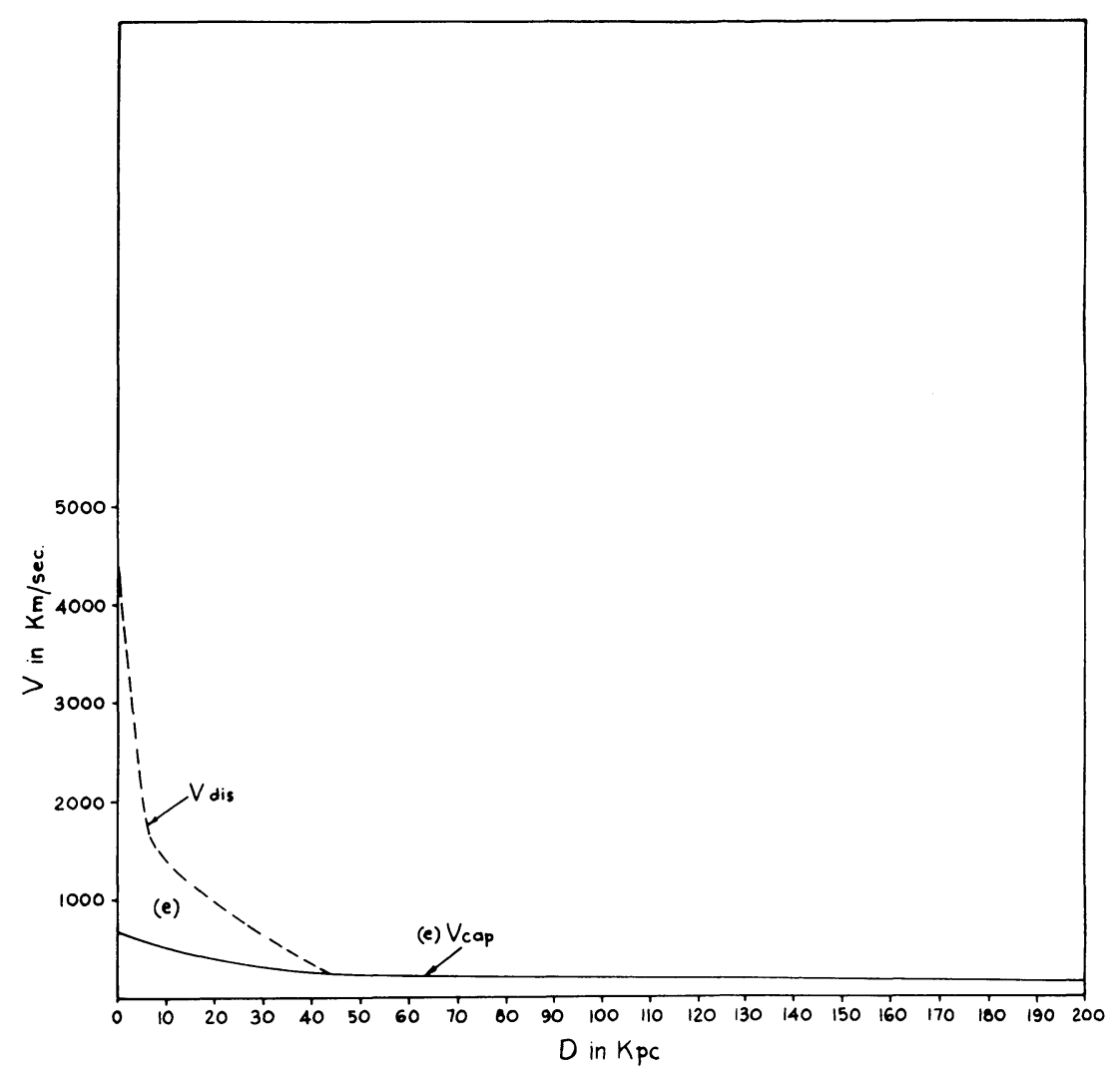

Fig. 4. The run of $V_{\text {cap }}$ and $V_{\text {dis }}$ with $D$ for case (e).

We conclude from the figures that formation of double galaxies by tidal capture without disruption of one of the galaxies is possible in a close collision between two galaxies of same dimension either if the two galaxies do not differ much in mass and density distribution or if the more massive galaxy is less centrally concentrated than the other. In cases (c), (d), and (e), $M_{2}$ will disrupt in close collisions for $V_{i}<V_{\mathrm{dis}}$. Disruption does not generally occur in distant collisions.

Cox and Toomre have studied the case of a head-on collision between two equal and approximately spherical galaxies presumed to have started from rest at infinity. Their results indicate that the tidal friction is so considerable that at least $80 \%$ of the total mass of the two galaxies soon tumbles into a single heap. (Toomre, 1974).

In order to study the effects of change in the dimensions of the galaxies on $V_{\text {cap }}$ we shall make use of the empirical statistical relationship between the potential energy and the mass of an elliptical galaxy obtained by Fish (1964):

$$
|\Omega|=9.6 \times 10^{-8} M^{3 / 2} \quad \text { (cgs units) }
$$

Comparing this with the potential energy of a polytrope of index $n=4$ (Chandrasekhar, 
1939), we have,

$$
M=0.23 R^{2} \quad \text { (cgs units) }
$$

We shall assume this relationship approximately and consider a giant galaxy to be of mass $10^{11} M_{\odot}$ and radius $10 \mathrm{kpc}$, and a dwarf galaxy to be of mass $10^{9} M_{\odot}$ and radius $1 \mathrm{kpc}$.

The curves (a), (g), and (h) in Figure 6 give the run of $V_{\text {cap }}$ with $D$ for giant-giant, giant-dwarf and dwarf-dwarf collisions respectively. The results indicate that the probability of the formation of double galaxies by tidal capture is greatest for giantgiant collisions and least for dwarf-dwarf collisions. Tidal disruption of a galaxy does not occur in a single collision between two typical globular galaxies differing in masses and dimensions in accordance with the adopted mass-radius relation. A galaxy may however disrupt if it undergoes more than one collision.

Following Rood's analysis (1965) closely for the determination of the number of captures in a cluster of galaxies from the values of $V_{\text {cap }}$ we find that, in a compact spherical cluster of galaxies of radius $1 \mathrm{Mpc}$, containing one thousand giant galaxies, having an isotropic Maxwellian distribution of velocities with velocity dispersion of

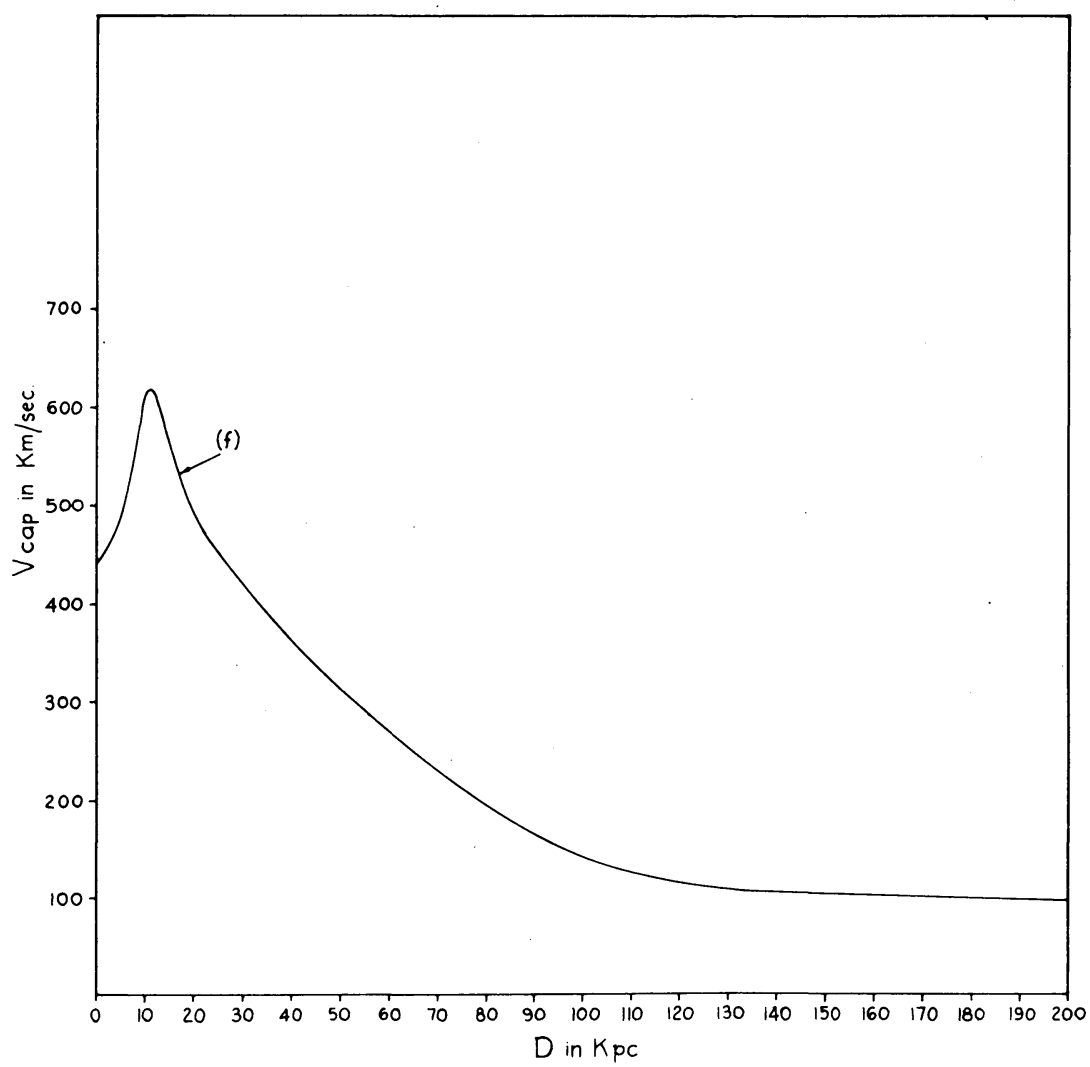

Fig. 5. The run of $V_{\text {cap }}$ with $D$ for case (f). 


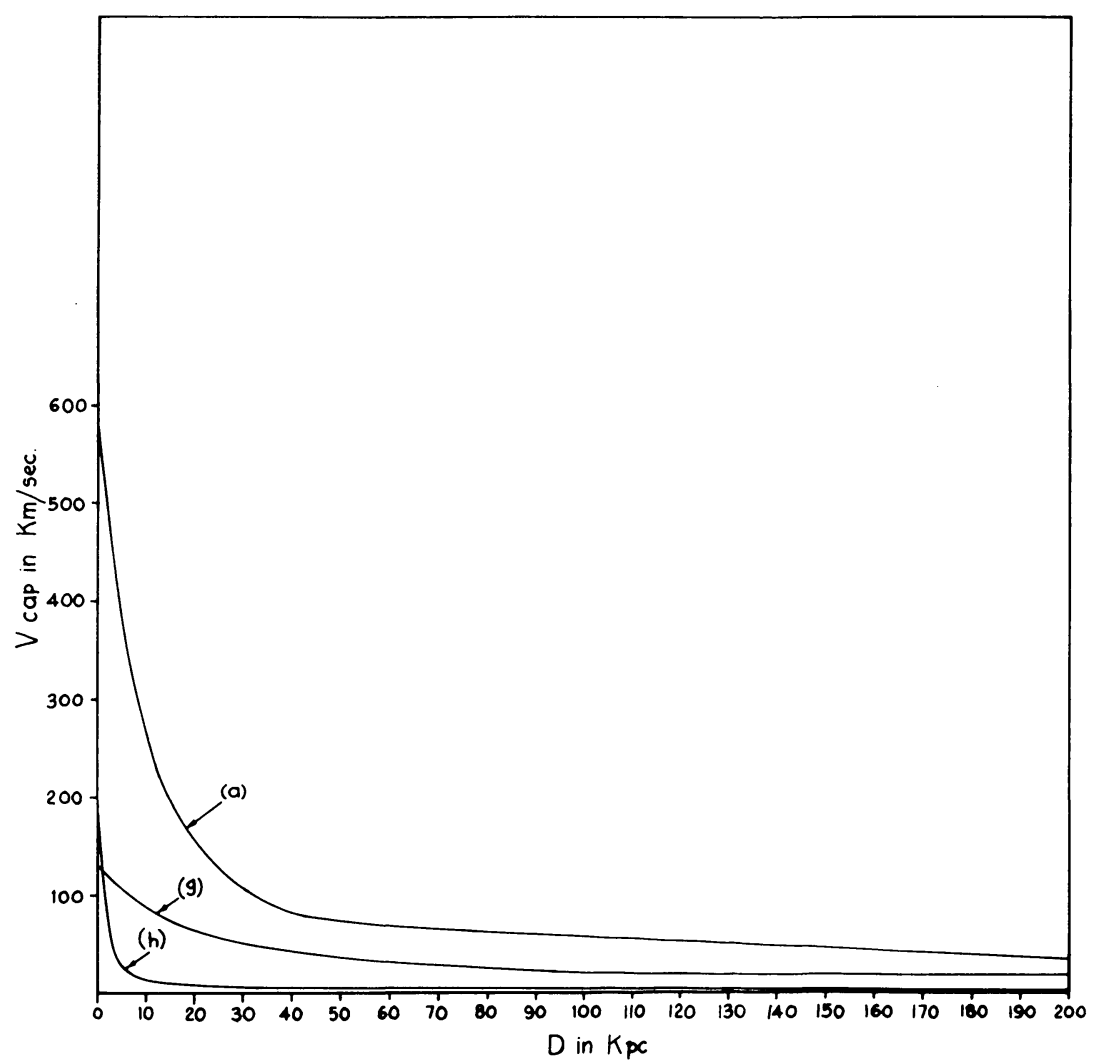

Fig. 6. The run of $V_{\text {cap }}$ with $D$ for (a) giant-giant collisions (g) giant-dwarf collisions, and (h) dwarf-dwarf collisions.

$500 \mathrm{~km} \mathrm{~s}^{-1}$ (this is the velocity required by the Virial theorem for stability), there will be about 2000 interpenetrating collisions in $10^{10} \mathrm{yr}$ and 20 of these will result in the formation of double galaxies by tidal capture. The Coma cluster is somewhat less compact than the model of the cluster considered and the velocity dispersion of the galaxies in the Coma cluster is about $1800 \mathrm{~km} \mathrm{~s}^{-1}$. Hence in the case of the Coma cluster the number of tidal captures is less by two orders of magnitude.

\section{References}

Alladin, S. M.: 1965, Astrophys. J. $141,768$.

Chandrasekhar, S.: 1939, An Introduction to the Study of Stellar Structure, University of Chicago Press, Chicago, Ch. 4.

Fish, R. A.: 1964, Astrophys. J. 139, 284.

Potdar, A. and Ballabh, G. M.: 1974, Astrophys. Space Sci. 26, 353.

Potdar, A.: 1974, 'Tidal Force Effects in Clusters of Galaxies', Osmania University (Ph.D. Thesis).

Rood, H. J.: 1965, 'The Dynamics of the Coma Cluster of Galaxies', University of Michigan (Ph.D. Thesis).

Sastry, K. S. and Alladin, S. M.: 1970, Astrophys. Space Sci. 7, 261. 
Sastry, K. S.: 1973, 'Change in the Gravitational Energy of Galaxies due to Collisions and its Consequences', Osmania University (Ph.D. Thesis).

Spitzer, L.: 1958, Astrophys. J. 127, 17.

Toomre, A.: 1974, in J. R. Shakeshaft (ed.), 'The Formation and Dynamics of Galaxies', IAU Symp. 58, 347.

\section{DISCUSSION}

Biermann: Don't your results let us understand very nicely why many clusters leave the two most massive galaxies form a binary ? Have the two massive galaxies a high probability of combining ? Isn't there many cluster galaxies where there are two very massive ones?

Alladin: The two massive galaxies do have a high probability of combining into a double system. This could be the reason why in clusters the two most massive galaxies form a binary.

Gott: The central binary pair of massive galaxies may be more probably formed via two body relaxation effects.

King: Will your captured pairs not continue to interact and spiral into each other?

Alladin: The double galaxies formed by tidal capture will revolve around each other with mean separations that will continually decrease in time. Ultimately the components of a double galaxy will form a single stellar system. I would guess that it would generally take time equal to two or three periods of revolution of the galaxies for a single system to form. Recent work by Cox and Toomre shows that the tidal friction in a slow head-on collision of galaxies is enormous.

Wielen: How is the loss of 'external' energy distributed within each galaxy? Does it somehow resemble the distribution of the gain in internal energy, which increases strongly with the distance of a star from the centre of its galaxy, or is it essentially constant over the whole galaxy?

Alladin: Due to the loss of external energy the relative motion of the centres of mass of the two galaxies will be retarded. It is conceivable that different parts of the two galaxies may be retarded at different rates. I think much computational labour would be needed to estimate this differential effect.

Innanen: Is it possible to computer simulate self-consistently the motion of two groups of say, 200 particles which have an encounter?

Aarseth: This problem is well within the reach of $N$-body calculations. Some simple calculations of colliding clusters with small particle numbers have already been made (Aarseth, S. J. and Hills, J. G.: 1972, Astron. and Astrophys. 21, 255).

Miller: Some years ago, in the early days of the big $N$-body calculation that gave spiral patterns we ran a problem in which two clumps of stars were allowed to fall together. The two were allowed to interpenetrate. After the collision there were two clumps moving apart, with a third clump at rest between the two, and some bridges connecting the two. The motion was not followed beyond this stage.

Van Albada: It should be kept in mind that the problem of penetrating galaxies is a collisionless one: what matters is the star-galaxy interaction. $N$-body calculations with $N \sim 200$ would suffer from star-star interaction.

Heggie: A student of Professor Hockney's has recently carried out computations, in three dimensions with some tens of thousands of stars, of the interaction between two galaxies. These are self-consistent calculations. 